Portland State University

PDXScholar

$1-1-2012$

\title{
Dynamic CCD pixel depletion edge model and the effects on dark current production
}

Justin Charles Dunlap

Portland State University

Morley M. Blouke

Portland State University

Erik Bodegom

Portland State University

Ralf Widenhorn

Portland State University

Follow this and additional works at: https://pdxscholar.library.pdx.edu/phy_fac

Part of the Physics Commons

Let us know how access to this document benefits you.

Citation Details

"Dynamic CCD pixel depletion edge model and the effects on dark current production," Justin C. Dunlap, M. M. Blouke, Erik Bodegom, Ralf Widenhorn, Proc. SPIE. 8298, 82980F (2012).

This Article is brought to you for free and open access. It has been accepted for inclusion in Physics Faculty Publications and Presentations by an authorized administrator of PDXScholar. Please contact us if we can make this document more accessible: pdxscholar@pdx.edu. 


\title{
Dynamic CCD pixel depletion edge model and the effects on dark current production
}

\author{
Justin C. Dunlap, M.M. Blouke, Erik Bodegom, and Ralf Widenhorn* \\ Portland State, Portland, OR 97207
}

\begin{abstract}
The depletion edge in Charge-Coupled Devices (CCD) pixels is dependent upon the amount of signal charge located within the depletion region. A model is presented that describes the movement of the depletion edge with increasing signal charge. This dynamic depletion edge is shown to have an effect on the amount of dark current produced by some pixels. Modeling the dark current behavior of pixels both with and without impurities over an entire imager demonstrates that this moving depletion edge has a significant effect on a subset of the pixels. Dark current collected by these pixels is shown to behave nonlinearly with respect to exposure time and additionally the dark current is affected by the presence of illumination. The model successfully predicts unexplained aspects of dark current behavior previously observed in some CCD sensors.
\end{abstract}

Keywords: Depletion edge, model, dark current, image correction, digital imagers, CCD, exposure time non-linearity, impurities

\section{INTRODUCTION}

Dark noise, a temperature dependent source of noise present in pixels of digital imagers such as charge-coupled devices (CCDs), is generated in an image whether or not the shutter is opened or closed. This source of noise is caused by electrons excited from the valence to the conduction band in the silicon comprising the pixels. A CCD's pixel architecture is similar enough to a p-n junction of a diode such that the pixels' dark current can be understood by studying this architecture. ${ }^{1-5}$ Along with lattice defects, the presence of heavy metal impurities such as $\mathrm{Ag}, \mathrm{Ni}$, and $\mathrm{Fe}$ within the pixels' silicon crystal's lattice are responsible for the non-uniform distribution of dark noise across the pixels in the imager. ${ }^{6-10}$

The amount of dark noise is generally expected to be about the same regardless of whether the imager is exposed to light or left in the dark. However, it was found in prior studies that the dark noise generation rate for certain pixels was changed by the presence of illumination. ${ }^{11}$ For these pixels, the standard method of removing this noise is insufficient. In particular, after an image is taken the standard method of correction involves taking an exposure at the same integration time as the image except now leaving the shutter closed. This "image" is referred to as a dark frame and, with the intention of removing the dark noise present in the original image, the pixel values from this dark frame are subtracted from the original image's pixel values. While more complex methods of correction exist, such as taking multiple dark frames and averaging them to reduce shot noise, they generally all rely on the expectation that the dark noise will be consistent when the shutter is open or closed.

We introduce a model that duplicates the changes to dark current generation rate by the imagers in the prior studies when exposed to light when compared to not being exposed. ${ }^{11}$ The model assumes the presence of one or more impurities in the pixel that produce a distinctive rate of dark current per impurity. Due to filling with signal charge the depletion edge of the potential well will shift possibly far enough to shift the impurity out of the well. Minimal amounts of dark current will therefore be collected from this impurity while no longer in the well. Light exposure essentially results in large amounts of signal charge being collected by the well and rapidly shifts the depletion edge. Therefore, it is possible for a pixel with one or more impurities in the region of the depletion edge shift to produce significantly less dark current when exposed to light than when not exposed.

*ralfw@pdx.edu

Sensors, Cameras, and Systems for Industrial and Scientific Applications XIII, edited by Ralf Widenhorn, Valérie Nguyen, Antoine Dupret, Proc. of SPIE-IS\&T Electronic Imaging, SPIE Vol. 8298, 82980F · () 2012 SPIE-IS\&T · CCC code: 0277-786X/12/\$18 · doi: 10.1117/12.909217 


\section{DYNAMIC DEPLETION EDGE MODEL}

\subsection{Model overview}

Figure 1 displays a schematic diagram of the moving depletion edge, located at a distance $w$ from the oxide layer, in a CCD pixel. An impurity, marked with an X in Fig. 1, will be located within the depletion region when the collection region contains no signal charge, however, as charge is collected the depletion edge moves and effectively shifts the impurity out of the collection region. The region of collection is taken to include the entire well from the surface to the depletion edge plus a small distance, $\delta$, where it is likely to collect a portion of the carriers created by an impurity within this region defined by $\delta$. Thermally generated electrons are still produced by impurities outside of the collection region. However, in this region the generation rate is much less than the depletion generation rate at assumed operating temperatures. In addition, it is conjectured that there will be a region where some of the generated dark current is lost when an impurity is located precipitously close to the depletion edge. For simplicity, this distance is assumed to be the same $\delta$.

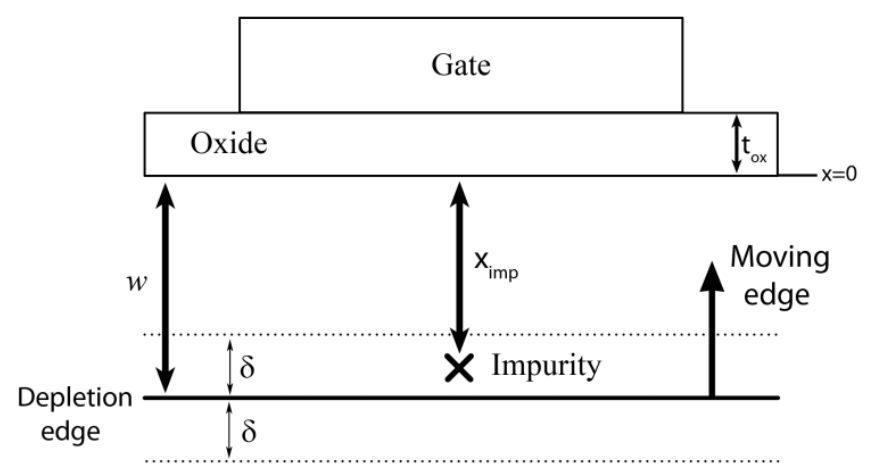

Figure 1- Moving depletion region in a CCD pixel

As signal charge is collected, the location of the depletion edge will change. Depletion edge depths are routinely calculated by employing impurity concentrations and fundamental electrostatics. ${ }^{1,2}$ We assume a Gaussian distribution of n-type donor atoms and follow the work of Taylor, et al. ${ }^{12}$ in calculating the depletion depth for a CCD pixel. The calculations are involved while providing minimal insight into the effects discussed in this work. They will be published elsewhere. It is sufficient to understand that the depletion edge will move closer to the oxide layer with increasing collected signal charge.

We employ the model to generate data to show an overview of the modeled pixels' dark current production when initially no signal charge exists (as in a traditional dark frame) and when initially there is a large amount of signal charge (such as a pixel would contain after being exposed to illumination) across the whole modeled imager. To do this, a statistical argument is used in assigning impurities to pixels similar to the methods of McGrath et al. ${ }^{6}$ If impurities are randomly distributed across the imager, the fraction of pixels, $P_{n}$, with a given number of impurities, $n$, can be calculated using a Poisson distribution such that:

$$
P_{n}=\left(\frac{N_{i m p}}{N_{p i x}}\right)^{n}\left[\frac{1}{n !} \exp \left(-\frac{N_{i m p}}{N_{p i x}}\right)\right],
$$

where $N_{i m p}$ is the total number of impurities present in the imager and $N_{p i x}$ is the total number of pixels on the imager. The number of pixels with $n$ impurities, $N_{n}$, is therefore $P_{n}$ multiplied by the total number of pixels, or:

$$
N_{n}=P_{n} N_{p i x} .
$$


Our model assumes a uniform distribution of impurities as a function of depth within the device and assigns a randomized depth to each impurity. The algorithm therefore creates $N_{n}$ pixels with $n$ impurities and assigns depths for each of the impurities within each pixel.

To calculate the dark current generated by a pixel with a given integration time, we first evaluate if all the impurities within that pixel will be located within the depletion area for the entire integration time. If so, the accumulated charge is calculated by multiplying the rate of dark current generation for the impurity, $R$, by the number of impurities and the integration time. This pixel will be unaffected by the shift in the depletion edge and will produce the same amount of dark current throughout an exposure with or without an initial exposure to light. However, if there is sufficient signal charge, either initially (due to a simulated exposure to light by initially filling the well with signal charge) or after collection of dark current for part of the integration time, to move the pixel's impurity outside of the depletion region, the generation rate for the pixel would not remain fixed at $R$. Therefore, the program iteratively calculates the quantity of charge accumulated per step size of the integration time, $\Delta t$, by calculating the depth of the depletion edge with the previously accumulated signal charge. Then, for every impurity within the pixel, a check is done to see if the impurity remains within the region of collection. The quantity of new charge collected due to the impurity, $\Delta q_{n}$, is modified by where the impurity is within the region of collection. There is still a statistical probability that these electrons will be collected by the pixel and this is the motivation for defining the region, $\delta$, as discussed previously, where a portion of the carriers created by an impurity just outside the depletion region will be collected by the pixel. There is also a statistical probability that some carriers generated by an impurity will be lost if the impurity is inside the depletion region but close to the depletion edge. Thus, the quantity of new charge within a distance plus or minus $\delta$, is approximated by calculating the collected charge in these regions using:

$$
\Delta q_{n}=R \Delta t \frac{\left(w-x_{i m p}\right)+\delta}{2 \delta}
$$

for each time step up until the end of the integration time. If the impurity is within the depletion region and further than a distance $\delta$ from the depletion edge, the new charge is calculated by multiplying the rate by $\Delta t$. If the impurity is not located within the well, there is no charge collected for that impurity during that time. The effects of varying $\delta$ are shown later.

To convert between charge $q_{s}$ and digital counts, $D$, we use the relation:

$$
q_{s}=\frac{D g}{A},
$$

where $g$ is the gain in $\mathrm{e}^{-} / \mathrm{DN}$ and $A$ is the collection area of the pixel.

The model first calculates a table of values relating depletion depth and signal charge based upon varying channel potentials. The model assigns depths and number of impurities within pixels and, using the aforementioned table, calculates the resulting dark current generated by those pixels for a given exposure time and initial signal count. The constants the model utilizes that can be thought of as dependent upon the camera system (and are therefore largely fixed) are the gate voltage, $V_{g}$, impact dose, $Q_{c d}$, oxide thickness, $t_{o x}$, number of acceptors per volume, $N_{a}$, relative oxide permittivity, $\varepsilon_{o x} / \varepsilon_{0}$, relative semiconductor permittivity, $\varepsilon_{S i} / \varepsilon_{0}$, range, $r$, straggle, $\sigma$, electron charge, camera gain, $g$, rate of dark current without impurity, $R_{0}$, rate of additional dark current due to a single impurity, $R_{l}$, pixel collection area, $A$, total pixels, $N_{p i x}$, full well count for a pixel, number of impurities, $N_{i m p}$, and $\delta$. Integration time and initial signal charge are variables that can be chosen by the user. Typical values were chosen to be in the range of those in common imagers and are given in Table 1. When using these values, the depletion edge starts at a distance of $5.33 \mu \mathrm{m}$ from the oxide layer and moves to $3.93 \mu \mathrm{m}$ when the depletion region is entirely filled with signal charge. 


\begin{tabular}{|c|c|}
\hline Variable & Value Chosen \\
\hline $\mathrm{V}_{\mathrm{g}}$ & $-5 \mathrm{~V}$ \\
\hline $\mathrm{Q}_{\mathrm{cd}}$ & $2.5 \times 10^{12} \mathrm{~cm}^{-2}$ \\
\hline $\mathrm{t}_{\mathrm{ox}}$ & $7.2 \times 10^{-6} \mathrm{~cm}^{-2}$ \\
\hline $\mathrm{N}_{\mathrm{a}}$ & $5.0 \times 10^{14} \mathrm{~cm}^{-2}$ \\
\hline$\varepsilon_{\mathrm{ox}} / \varepsilon_{0}$ & 3.9 \\
\hline$\varepsilon_{\mathrm{Si}} / \varepsilon_{0}$ & 11.7 \\
\hline $\mathrm{r}$ & $1.0 \times 10^{-5} \mathrm{~cm}$ \\
\hline$\sigma$ & $3.0 \times 10^{-5} \mathrm{~cm}$ \\
\hline $\mathrm{g}$ & $3.0 \mathrm{e}-/ \mathrm{DN}$ \\
\hline $\mathrm{R}_{0}$ & $1.0 \mathrm{DN} / \mathrm{s}$ \\
\hline $\mathrm{R}_{1}$ & $10.0 \mathrm{DN} / \mathrm{s}$ \\
\hline $\mathrm{A}$ & $2.1 \times 10^{-7} \mathrm{~cm}$ \\
\hline $\mathrm{N}_{\mathrm{pix}}$ & $1 \times 10^{6}$ \\
\hline Full well count & $65,536 \mathrm{DN}(16 \mathrm{bit})$ \\
\hline $\mathrm{N}_{\text {imp }}$ & $50,000 \mathrm{impurities}$ \\
\hline$\delta$ & $5.0 \times 10^{-6} \mathrm{~cm}$ \\
\hline Integration time & $500 \mathrm{~s}$ \\
\hline Initial signal charge & $30,000 \mathrm{counts}$ \\
\hline
\end{tabular}

Table 1- Typical values used by the model

\subsection{Modeled response to illumination}

To get an overview of the effect of illumination, or an initial partial filling of the potential well, we define $\Delta D_{\text {Light }}$ :

$$
\Delta D_{\text {Light }}=D_{N L}-D_{L},
$$

where $D_{N L}$ is the total number of dark counts generated with no initial signal charge in the potential well for a given integration time, and $D_{L}$ is the total number of dark counts generated for the same integration time after the initial flash.

A two-dimensional distribution of the loss in dark count, $\Delta D_{\text {Light }}$, versus the dark-count level, $D_{N L}$, was created. The $\Delta D_{\text {Light }}$ versus $D_{N L}$ plane was divided into intervals of size $250 \times 250 \mathrm{DN}$ and the shades of gray represent the logarithm of the number of pixels in a specific interval.

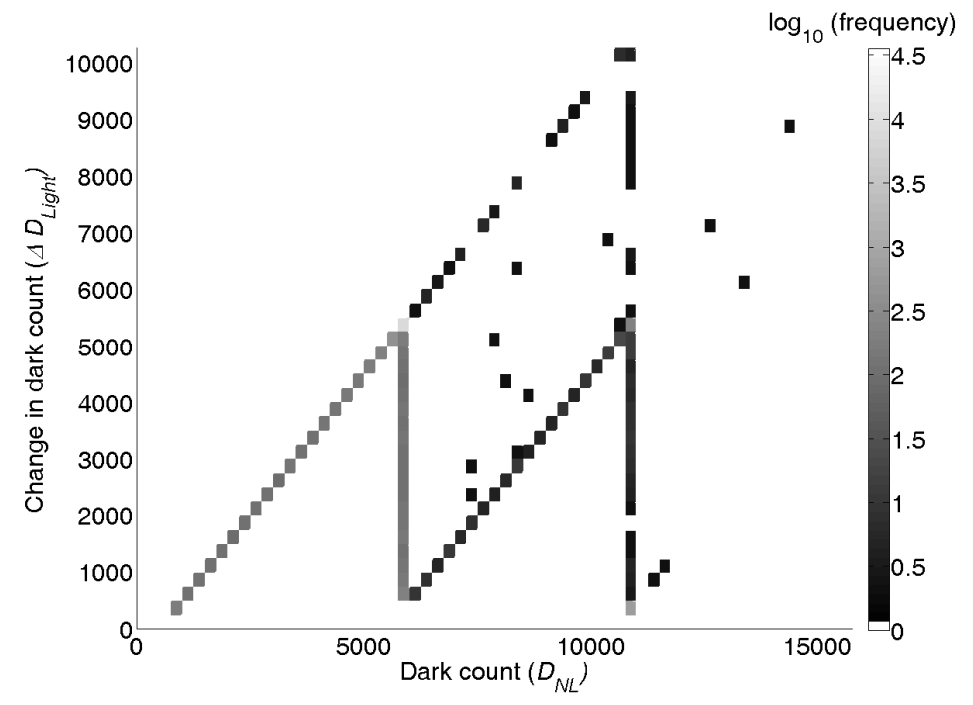

Figure 2- Change of dark count after illumination versus dark count of a $500 \mathrm{~s}$ dark frame with an initial count of 30,000 . 
A pixel with a single impurity that remains fully within the depletion region for the entire collection time without exposure to light will have a $D_{N L}$ of about $5,500 \mathrm{DN}$. If additionally the impurity remains fully within the depletion region during the collection time after the initial flash, the impurity will produce just as much dark current and will have a $\Delta D_{\text {Light }}$ value of about 0 . However, if the initial flash of light moves the depletion edge past the impurity, the pixel will collect no dark current from the impurity and the pixel will have a $\Delta D_{\text {Light }}$ of about 5,500 . If the depletion edge moves past the impurity at some point during the collection time after the exposure of light, the pixel will lie in the grouping of pixels parallel to the y-axis at about 5,500 DN. Now, if the impurity lies close enough to the depletion edge such that it is partially or fully removed from the depletion region during the collection time without exposure to light, the pixel will have a $D_{N L}$ value less than $5,500 \mathrm{DN}$. Of course, the impurity will also be removed from the depletion region by the exposure to light or during the subsequent collection time after the exposure so the pixel will have a small or zero $D_{L}$ value and $\Delta D_{\text {Light }}$ will be about the same value as $D_{N L}$.

A pixel with two impurities which remain fully within the depletion region during the collection time with no exposure to light will have a $D_{N L}$ of about $11,000 \mathrm{DN}$. If both those impurities are also fully within the depletion region for the entire collection time after the flash, the pixel will again produce just as much dark current and will have a $\Delta D_{\text {Light }}$ value of about 0 . If both impurities are fully removed from the depletion region by the flash, the pixel will produce no dark current for the collection period giving a $D_{L}$ value of 0 and $\Delta D_{\text {Light }}$ will also be about $11,000 \mathrm{DN}$. If just one impurity is removed from the collection region by the flash while the other remains fully within the region for the entire collection time, the $D_{L}$ value will be about 5,500 and thus the $\Delta D_{\text {Light }}$ value will also be about 5,500. If one or both impurities are removed from the collection region during the collection time after the flash, the pixel will lie in the grouping parallel to the y-axis. For the cases where one or both impurities are partially removed from the collection region during the collection time with no exposure to light $D_{N L}$ will have values less than 11,000 DN. During or after the flash, if one impurity stays fully within the collection region while the other is removed, $D_{L}$ will have a value between 5,500 and 11,000 , and therefore $\Delta D_{\text {Light }}$ will be between 0 and 5,500, approximately the same as $D_{N L}$ for that pixel. If both pixels are removed from the depletion region during or after the flash, $D_{L}$ will be between 0 and 5,500. If both impurities are turned off during the flash, $\Delta D_{\text {Light }}$ will be approximately the same as $D_{N L}$. However, if one of the impurities is removed from the depletion region only in the collection time after the flash, the pixel will lie between the two diagonal lines with a $D_{N L}$ value between 5,500 and 11,000.

Additionally, pixels with more impurities of the type we have modeled are possible but become statistically unlikely with increasing impurities. There are few pixels with three impurities which can be seen in Fig. 2 with $D_{N L}$ values greater than 11,000 .

\section{DISCUSSION}

The observed results from the model correspond to many features seen in similar plots of experimental data for existing cameras. Two cameras displaying these behaviors are the SBIG ST-8XE with a KAF-1602E CCD sensor and a Meade Pictor 416XT with a Kodak KAF-0400 CCD chip. A previous publication ${ }^{11}$ included figures similar to those in Fig 2, where exposures were taken to highlight the effects discussed above. Using the settings listed in Table 1, except for as detailed in this paragraph, the model was used to generate graphs matching the actual experimental data. The KAF$1602 \mathrm{E}$ has $1.56 \times 10^{6}$ pixels and a gain of $2.3 \mathrm{e}-/$ count, while the KAF- 0400 has $3.93 \times 10^{5}$ pixels and a smaller gain of $1.2 \mathrm{e}-/ \mathrm{count}$; these values were used for $N_{p i x}$ and $g$ respectively. Based on dark current production for a standard pixel with very little dark current production $R_{0}$ was taken to be $0.5 \mathrm{DN} / \mathrm{s}$ for both imagers and based on dark frame histograms for the peak of hot pixels in the imager, we used $9.5 \mathrm{DN} / \mathrm{s}$ for $R_{l}$ for the KAF-1602E and $9.0 \mathrm{DN} / \mathrm{s}$ for the KAF-0400. $N_{i m p}$ was chosen to be 20,000 impurities for the KAF-1602E and 50,000 impurities for the KAF-0400. These values were chosen to generate approximately the same number of pixels as seen in the peak of pixels at about 6,000 DN in Fig 3a and 4a respectively. A $\delta$ value of $3.0 \times 10^{-6} \mathrm{~cm}$ provided for the best fit for the groupings for the KAF-1602E and a value of $5.0 \times 10^{-6} \mathrm{~cm}$ provided for the best fit for the groupings for the KAF-0400. This value is empirically chosen for the two imagers and our model does not attempt to explain the differing values; they may depend on such factors as the doping concentration. For the graphs from the previous publications, groupings were boxed and labeled for the ease of discussion and we have left the boxes in these figures as well as superimposed them in the modeled data for comparison. 


\subsection{SBIG ST-8XE}

Shown in Fig. 3a is the change in dark count after illumination versus dark count of a $600 \mathrm{~s}$ dark frame after an exposure to light leading to an average value of $26,000 \mathrm{DN}$ across the imager. These values were chosen as the integration time and initial signal respectively for the modeled data seen in Fig. 3b.
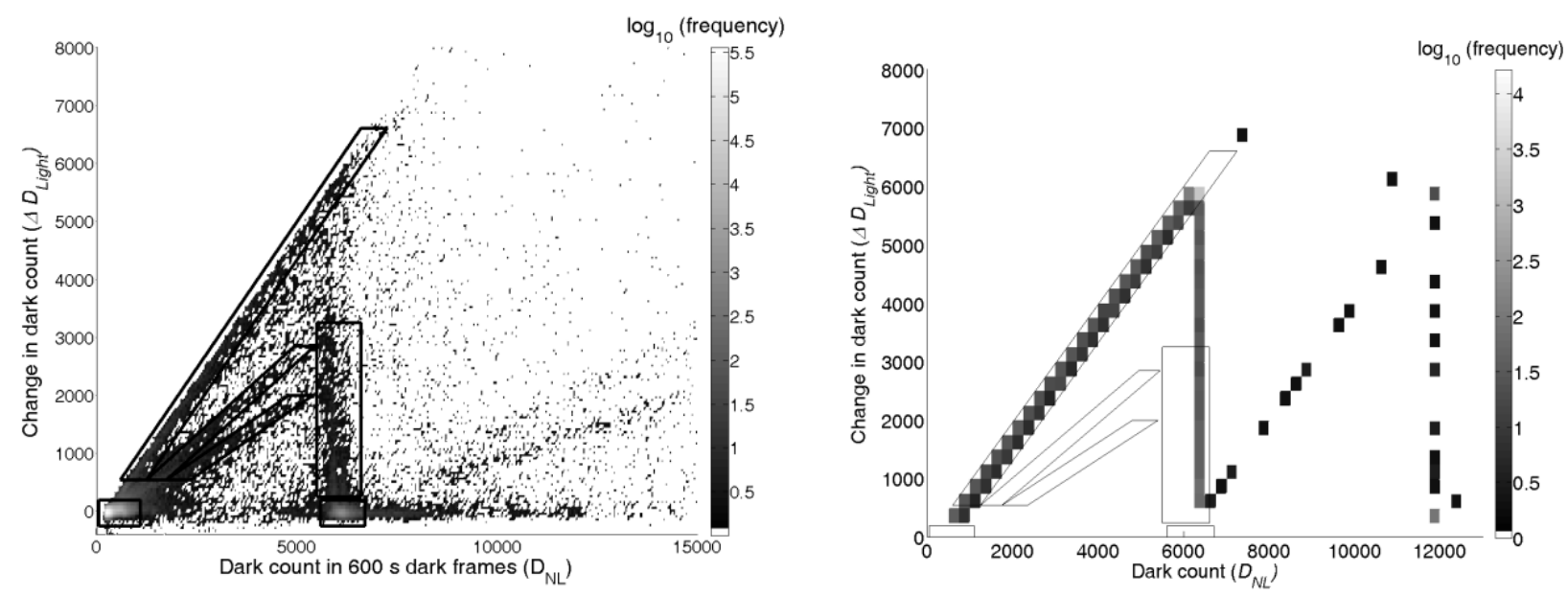

Figure 3: Change of dark count after illumination versus dark count of a $600 \mathrm{~s}$ dark frame.

(a) SBIG ST-8XE for an average initial count due to the light exposure of approximately 26,000 at 288

K. ${ }^{11}$ (b) modeled data.

For many of the features, there is agreement between experimental and modeled data. In particular, the major groupings of pixels seen at a dark count of about 6,000 in Fig. 3a, those seen in the horizontal groupings above these values, and those seen at a diagonal connected to the top of those horizontal groupings, are all present in the experimental as well as the modeled data. All of these pixels can therefore be explained by a pixel having a particular type of impurity that generates dark current at a rate of about $9.5 \mathrm{DN} / \mathrm{s}$. The location of the pixel in the graphs will depend on what the depth of the impurity is compared to the quantity of signal charge accumulated in the various exposures used to create the graphs. In addition, there is evidence in the experimental data of groupings seen due to pixels with two impurities including the extension of the diagonal grouping past the horizontal grouping at about $10 \mathrm{DN} / \mathrm{s}$.

While not sufficient to explain all of the features seen in the experimental data, such as the groups of pixels off the main diagonals (with the greatest slope) and the specific number of pixels seen in each of these groupings, many of the features are captured. The deficiencies of the model are likely due to the model being essentially only one-dimensional whereas a more complete model would require analyzing the change in the shape of the three-dimensional depletion region. In addition, our model assumes a uniform distribution of impurities as a function of depth within the pixel, however there may be processes that result in a more systematic location of impurities. A larger percentage of impurities within the region of the depletion edge variation will result in a greater number of pixels in the labeled groups in the experimental data. In addition, a three-dimensional model in which the depletion edge moves significantly more near the edges of the pixel may result in more pixels affected by a similar type of nonlinearity. One possible explanation for more impurities in this region of greater change, would be impurities introduced in building the channel stops. Inclusion of these considerations is an area for future work. 


\subsection{Meade Pictor 416XT}

Shown in Fig. 4a is a similar graph for the Meade imager system, where the change in dark count after illumination versus dark count of a $600 \mathrm{~s}$ dark frame after an exposure to light leading to an average value of 42,000 DN across the imager. These values were chosen as the integration time and initial signal respectively for the modeled data seen in Fig. $4 b$.
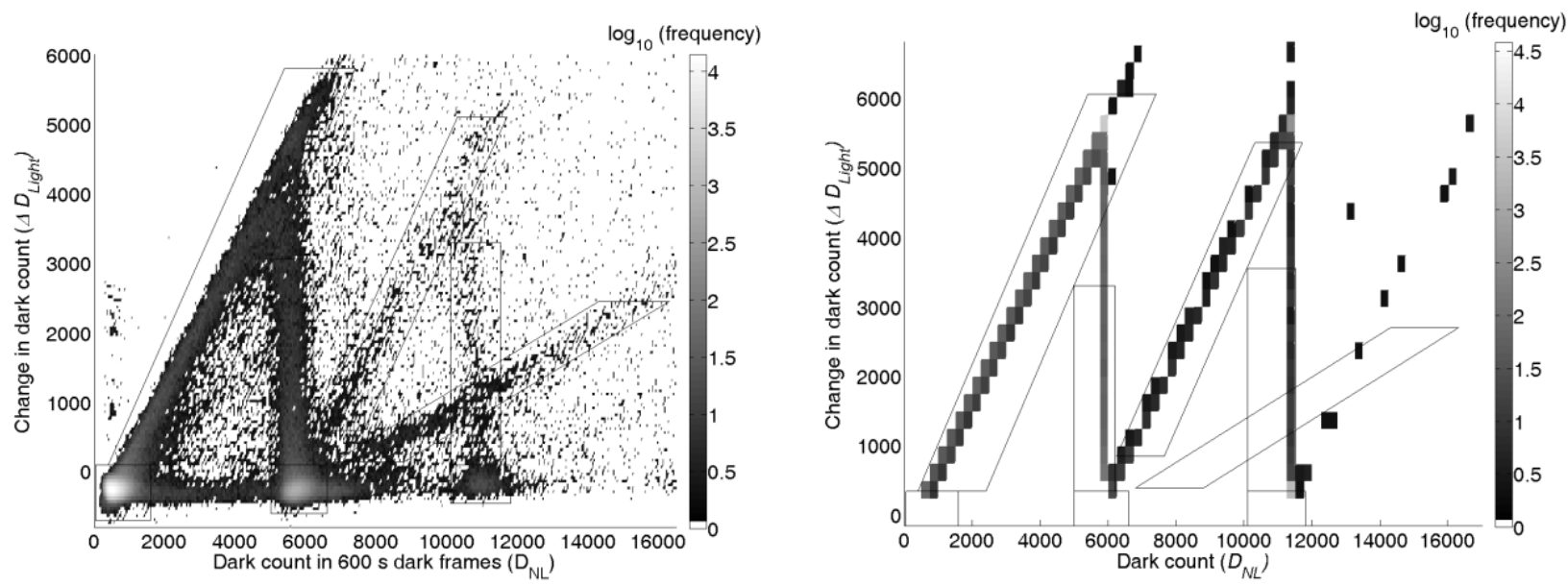

Figure 4: Change of dark count after illumination versus dark count of a $600 \mathrm{~s}$ dark frame.

(a) Meade Pictor 416XT for an average initial count due to the light exposure of approximately 42,000 at $278 \mathrm{~K}^{11}{ }^{11}$ b) modeled dark current.

Again, agreement in the features is seen in the graphs of the experimental and modeled data. Due to the relatively larger number of impurities seen in the KAF-0400, there is a second triangle of data seen with larger dark counts along the $\mathrm{x}$ axis. This is due to more pixels that have a higher likelihood of having two impurities. Thus, all of the features seen in Fig. $4 \mathrm{~b}$ can be explained by a pixel having one or more impurities with a rate of production at $9.0 \mathrm{DN} / \mathrm{s}$. There exists a grouping starting at about 6,000 counts and $0 \Delta D_{\text {Light }}$ that extends diagonally in the graph to 18,000 counts and a $\Delta D_{\text {Ligh }}$ of about 3,000 that is not explained by the model. This grouping shares similarities with the unexplained grouping in the SBIG 8XE imager.

\section{CONCLUSION}

Dark noise generation rates that change with exposure to illumination compared to no exposure can be reproduced using a model of a one-dimensional depletion well with a moving depletion edge and containing one or more impurities near the edge. Control of the variables in the model allows for accurately predicting the amount of dark current generated by a pixel as the depletion well is filled with signal charge. The distinctive patterns and groupings seen in multiple camera systems when dark current generation rates are compared in the presence or absence of light are duplicated by this model.

\section{REFERENCES}

[1] Sze, S.M., [Physics of Semiconductor Devices], second edition (John Wiley \& Sons, (1981).

[2] Grove, A.S., [Physics and Technology of Semiconductor Devices], (John Wiley \& Sons (1967).

[3] Hall, R.N., "Electron-hole recombination in germanium," Phys. Rev. 87, 387-388 (1952).

[4] Sah, C.T., Noyce, R.N., and Shockley, W., "Carrier generation and recombination in p-n junction and p-n junction characteristics," Proc. IRE, 45 1228-1248, (1957).

[5] Shockley, W., and Read, W.T., "Statistics of the recombination of holes and electrons," Phys. Rev. 87 835-842 (1952).

[6] McGrath, R.D., Doty, J., Lupino, G., Ricker, G., and Vallerga, J., "Counting of deep-level traps using a chargecoupled device," IEEE Trans. Electron Devices, vol. ED-34, 2555, (1987).

[7] Widenhorn, R., Blouke, M.M., Weber, A., Rest, A., and Bodegom, E., "Temperature dependence of dark current in a CCD,” Proc. SPIE Int. Soc. Opt. Eng. 4669, 193 (2002). 
[8] McColgin, W.C., Lavine, J.P., Kyan, J., Nichols, D.N., and Stancampiano, C.V., "Dark current quantization in CCD image sensors" International Electron Device Meeting 1992, 113, 13-16 (1992).

[9] McColgin, W.C., Lavine, J.P, and Stancampiano, C.V., "Probing metal defects in CCD image sensors," Mater. Res. Soc. Symp. Proc. 378, 713, (1995).

[10] McColgin, W.C. Lavine, J.P., Stancampiano, C.V., and Russell, J.B., "Deep-level traps in CCD image sensors" Mat. Res. Soc. Symp. Proc. 510, 475, (1998).

[11] Widenhorn, R., Hartwig, I., Dunlap, J.C., and Bodegom, E., "Influence of illumination on dark current in chargecoupled device imagers," Journal of Electronic Imaging, 18(3), (2009).

[12] Taylor, G.W., Chatterjee, P.K., and Chao, H.H., "A device model for buried-channel CCD's and MOSFET's with Gaussian impurity profiles,” Electron Devices, IEEE Transactions on, vol.27, no.1, 199- 208 (1980). 